\title{
Correlación entre embriocardia, saco vitelino y cuerpo lúteo en emba- razo menor de diez semanas para evaluar su viabilidad y evolución
}

\author{
Jimmy Castañeda*; Mauricio Rojas**; Andrés Sarmiento***; Vicente Carmona****
}

\begin{abstract}
RESUMEN: Existen reportes en la literatura mundial que, considerando algunos parámetros de ecografía temprana, hacen predicciones sobre la evolución del embarazo. Se pretendió demostrar estos hallazgos reportados universalmente para su aplicabilidad a la población que consulta al Hospital Militar Central, para la cual se realizaron ecografías transvaginales a 220 pacientes con embarazos menores de 10 semanas, en la Unidad de Ecografía Ginecoobstétrica del Hospital Militar Central o en la Unidad de Diagnóstico obstétrico y Ginecológico, (Obgyn), midiendo el diámetro del saco vitelino, cuantificando la embriocardia y valorando la presencia del cuerpo lúteo. Se realizó el seguimiento de las pacientes al final del período de estudio. Fueron excluidas 56 pacientes y con las 164 restantes no fue posible corroborar lo reportado en la bibliografía. Por tal motivo no podemos hacer ninguna predicción sobre el pronóstico del embarazo basados en las mediciones de ciertos parámetros por ecografía transvaginal en embarazos tempranos.
\end{abstract}

PALABRAS CLAVES: Embarazo temprano, ecografía transvaginal, pronóstico del embarazo.

SUMMARY: There are wordwide many medical reports about the pronostic value of some early pregnancy sonographic parameters on endovaginal ultrasound, such as cardiac pulsations, yolk sac diameter and the corpus luteum. This paper intends to evaluate the findings and to look for the possibility of use them as a predictor of pregnancy outcome in patients who assist to prenatal control at the Central Military Hospital of Bogota. We study 220 patients who were less that 10 weeks pregnant, who assisted to prenatal control at the Ultrasound Unit of the Obstetric and Gynecology Unit of the Central Military Hospital or to the Obstetric and Gynecological Diagnosis Unit (Obgyn). All of them underwent endovaginal sonography and had a determination of yolk sac diameter, cardiac pulsations and determination of the presence of the corpus luteum. We prospective followed 164 patients but we could not find what the literature says. The results of the study do not let us state that some endovaginal ultrasound parameters are an early predictor of pregnancy outcome.

KEY WORDS: Early pregnancy, endovaginal ultrasound, pregnancy outcome.

Teniendo en cuenta que algunos investigadores han demostrado que solamente el $57 \%$ de todas las concepciones van más allá de la semana 20 de gestación se puede considerar la reproducción humana como un proceso relativamente ineficaz. Del total de pérdidas, el $75 \%$ se produce antes de la implantación y sólo se reconocen clínicamente un $25 \%$ (1).

El riesgo de aborto en una mujer sin antecedentes de errores en la reproducción es de aproximadamente el $15 \%$, riesgo este que aumenta en los casos en los cuales la mujer tiene antecedentes de dos o más abortos (1-2). Más de la mitad de estas pérdidas tienen por causa defectos genéticos, y se dice que hasta un $20 \%$ son morfológicamente anormales (2).

A través del tiempo se han tratado de identificar ciertos parámetros, ecográficos así como de medidas de laboratorio, para predecir la evolución del embarazo, de manera tal que cuando se realicen en la fase inicial del mismo, puedan permitir una intervención terapéutica,

\footnotetext{
* Instructor Sección Ginecología y Obstetricia. Hospital Militar.

** Residente Nivel IIl, Sección Ginecología y Obstetricia. Hospital Militar Central.

*** Especialista Ginecología y Obstetricia. Unidad de Diagnóstico de Ginecología y Obstetricia (Obgyn).

**** Jefe Unidad de Ecografia y de Medicina Materno Fetal. Sección Ginecología y Obstetricia. Hospital Militar Central. Universidad Militar Nueva Granada. Escuela Militar de Medicina. Hospital Militar Central. Santafé de Bogotá, Colombia.
}

logrando así, mejorar los resultados. Infortunadamente se han reportado estudios que no han logrado una alta correlación entre los hallazgos ecográficos tempranos y las causas de abortos recurrentes (4).

El saco vitelino primitivo se forma aproximadamente en el día 23 de edad gestacional contada a partir del último período menstrual. En este momento el producto total de la concepción tiene aproximadamente $0.1 \mathrm{~mm}$ de diámetro. El saco vitelino secundario se sucede en el día 27 a 28 de edad gestacional, y en ese momento alcanza un diámetro de $3 \mathrm{~mm}$. Este saco vitelino puede ser evidenciado por ecografía transvaginal en forma fácil en etapas precoces del embarazo, puesto que hay ciertos parámetros que son identificables: primero, volumétricamente es mayor que un embrión; y segundo, es un saco que contiene líquido, lo cual le da un contraste relativamente alto (8). La visualización del saco vitelino siempre precede a la visualización del embrión mientras que su no visualización se ha asociado en un $48 \%$ a abortos que se presentan en corto tiempo, (7). Una vez que el embrión es visible, y su embriocardia cuantificada, la importancia en la identificación del saco vitelino disminuye (6).

El embrión se convierte de una estructura discoide trilaminar a una estructura más tubular, aproximadamente en el día 40. Mediante los procedimientos ecográficos rutinarios, transvesicales, Ia identificación del saco gestacional no es factible sino hasta la semana 5 o 5.5 , mientras que el eco embrionario sólo se logra detectar en la sexta semana (3). 
Durante el período embrionario, la identificación ecográfica de la actividad cardíaca es el principal deter-. minante para considerar la viabilidad futura del embarazo. Aún así, la visualización de un saco gestacional sin un embrión en su interior hace difícil la diferenciación entre un embarazo viable y uno no viable. El manejo tradicional incluye el seguimiento seriado de dicho saco, lo cual puede tomar una o incluso dos semanas, puesto que el crecimiento nulo o escaso del saco, indican, bien sea un aborto retenido o un embarazo anembrionado; la tardanza en la definición del diagnóstico está aumentando el riesgo de morbilidad y la ansiedad de las pacientes (3).

La determinación del tamaño mínimo en el cual se logra la identificación del saco vitelino es motivo de controversia, pero en términos generales se acepta un tamaño aproximado de 7 a $8 \mathrm{~mm}$, momento a partir del cual empieza a disminuir, cuando la medición se realiza transvesical, aunque si la valoración se realiza transvaginal, puede detectarse antes. Así mismo, el saco vitelino debe estar presente en embarazos normales por encima de cierto tamaño del saco gestacional, que se ha definido en $8 \mathrm{~mm}$ por ecografía transvaginal y en $20 \mathrm{~mm}$ por ecografía transvesical (9).

El tamaño del saco vitelino no ha sido el único parámetro al evaluar esta estructura, puesto que la deformidad de las paredes, debido a pérdida en la tensión de la misma, se había considerado como un parámetro diagnóstico definitivo de mala evolución, pero se tienen reportes de varios sacos deformados, que a la postre dieron como resultado embarazos con un desarrollo dentro de límites normales. Por lo tanto un saco deformado, aunque es fuertemente un indicador de no viabilidad, no es un signo conclusivo de la misma y la ausencia de otros parámetros de mal pronóstico debe ser interpretada con cautela (10).

La actividad cardíaca del embrión puede visualizarse por vez primera el día 34 luego del último período menstrual y debe ser observada en el día 37 en los embarazos normales (11). La frecuencia cardíaca del embrión aumenta de manera progresiva hasta el día 63 o cuando el embrión tiene $22 \mathrm{~mm}$ de longitud; pero el punto máximo se alcanza en el momento cuando se ha completado la formación del corazón del embrión, teniendo el aumento de la frecuencia un crecimiento paralelo con el desarrollo del corazón embrionario. La disminución de la frecuencia cardíaca se correlaciona con un mal pronóstico del embarazo, ya que se ha visto que los embriones cuya frecuencia cardíaca empieza a disminuir, terminarán siendo abortados (12). El mayor número de pérdidas se ha observado cuando el valor de la embriocardia está por debajo de 90 antes de la semana diez (10), mientras que cuando se obtienen valores menores a 70 , todos los embriones son abortados (13).

La concentración de progesterona ha sido determinada para cada una de las semanas del embarazo, así como su patrón de elevación, conociéndose que la hormona tiende a triplicarse entre la tercera y la treceava semana, aunque realmente no se observan elevaciones significativas entre las semanas quinta a décima (16).

El criterio ecográfico más importante en la determinación del pronóstico del embarazo en el primer trimestre, es la presencia o ausencia de un embrión cuya embriocardia sea visible, mientras que la presencia o ausencia de un saco vitelino parece no tener el mismo impacto diagnóstico y no puede ser usado como parámetro exclusivo para determinar si el embarazo es anormal o no. El cuerpo lúteo no siempre se visualiza en embarazos normales y su ausencia no siempre es indicador de mal pronóstico (6).

Teniendo en cuenta los datos anteriores, es probable que: 1) El volumen del cuerpo lúteo en la fase inicial del embarazo humano debe correlacionarse con la concentración de la progesterona producida por éste; 2) La apariencia del cuerpo lúteo, basado en la cantidad de quistes que posea, podría correlacionarse con la concentración hormonal o con el pronóstico del embarazo; y 3 ) Una disminución del volumen del cuerpo lúteo podría estar en relación con una pérdida precoz del embarazo. Aún así, hay estudios que no han podido comprobar estas hipótesis (17).

\section{Materiales y métodos}

Teniendo en cuenta el número total de pacientes que se estudian mediante ecografía en el Hospital Militar Central, (aproximadamente 600 al año) se considera adecuada utilizar una muestra de 150 pacientes para la investigación, tamaño muestral que nos permite interpretar información con un nivel de confianza del $95.5 \%$ y un margen de error de $\pm 7.1 \%$ según la estadística muestral:

Se realizó un estudio observacional prospectivo con 220 pacientes con embarazos menores de diez (10) semanas, que asistieron a consulta ecográfica en la unidad de ultrasonido del servicio de Ginecología y Obstetricia del Hospital Militar Central, o a consulta en el Instituto de diagnóstico obstétrico y Ginecológico (Obgyn), sin importar ningún tipo de antecedente. El rango de edad fue de 19 a 42 años.

Se realizaron exploraciones ecográficas transvaginales a las pacientes objeto de estudio con un equipo Siemmens Sonoline SL2, en las cuales se determinaron los tamaños del cuerpo lúteo, saco gestacional y saco vitelino, así mismo se cuantificó la embriocardia. Se hizo un seguimiento a las pacientes mediante revisión de la historia clínica y/o contacto telefónico para determinar la evolución del embarazo, considerando como viable todo embarazo que sobrepasara las 28 semanas. Se indagó sobre la presencia de patologías asociadas a la gestación. El período de recolección de datos fue del 01 de julio de 1995 a 30 de octubre de 1996, y el seguimiento final a las pacientes se realizó en la última semana de diciembre de 1996.

\section{Resultados}

De las 220 pacientes que fueron valoradas inicialmente fueron excluidas 56 por no haberse podido realizar el seguimiento hasta el final del embarazo o porque en el momento de la valoración inicial no se logró la recolección de todos los datos, o porque la final del estudio no fue posible la revisión de la historia clínica ni se logró contacto telefónico con la paciente. Se incluyen en el estudio aquellas pacientes en quienes no se visualizó el cuerpo lúteo ya que hay que tener presente que su visualización no es factible sino en un porcentaje de pacientes que incluso no alcanza al $50 \%$. 
La edad gestacional se calculó tomando como base la medición del saco gestacional o la medición de la longitud coronilla nalga, y este criterio primó sobre la edad gestacional calculada con base en la fecha de la última menstruación.

Las patologías halladas al inicio del estudio fueron: amenaza de aborto $15.2 \%$, presencia de dispositivo intrauterino $1.2 \%$, presencia de saco gestacional irregular $1.8 \%$ y otro de tipo de alteraciones $2.4 \%$. El resto de pacientes examinadas, $79.3 \%$ no presentaban alteraciones. Al final del embarazo las patologías encontradas fueron: Amenaza de aborto $11.0 \%$, amenaza de parto pretérmino $5.9 \%$ hipertensión inducida por el embarazo $1.7 \%$, retardo del crecimiento intrauterino $1.7 \%$ y otras patologías $0.8 \%$. De igual manera $78.8 \%$ de los embarazos llegaron a término sin presentar patología alguna.

Las pacientes que presentaron amenaza de aborto tuvieron una cuantificación de la embriocardia que varió entre 120 y 197 latidos por minuto. En el caso de amenaza de parto pretérmino las variaciones de la embriocardia estuvieron entre 125 y 185 latidos por minuto. Para las otras patologías la embriocardia osciló entre 111 y 195 latidos por minuto. El resto de pacientes, 133, que no presentaron alteraciones en el embarazo tuvieron una embriocardia que varió entre 101 y 205 latidos por minuto. Hay que anotar que en el presente estudio ninguna de las pacientes presentó embriocardia menor de 100 latidos por minuto.

El cuerpo lúteo se visualizó en sólo 50 pacientes y de ellas 42 no presentaron ninguna alteración en el embarazo. De las otras 8 pacientes 3 presentaron amenaza de parto pretérmino y 3 amenaza de aborto. Pero de las 118 a quienes no se les visualizó el cuerpo lúteo 12 presenta- ron amenaza de aborto y 7 amenaza de parto pretérmino. La incidencia de hipertensión inducida por el embarazo y de retardo de crecimiento intrauterino fue similar: 2 pacientes para cada patología.

El tamaño del saco vitelino que se consideró como límite normal, teniendo en cuenta lo reportado en la literatura mundial, fue de $6.0 \mathrm{~mm}$. Pero se tuvieron embarazos normales con sacos vitelinos tan pequeños como 2.4 $\mathrm{mm}$ o tan grandes como $8.5 \mathrm{~mm}$. Al correlacionar las mediciones del saco vitelino y la presencia de patologías se pudo observar que para la amenaza de parto pretérmino la medición del saco vitelino varió entre 3.0 y $6.7 \mathrm{~mm}$. Para los casos de amenaza de aborto la medición osciló entre 1.0 y $6.8 \mathrm{~mm}$, mientras que para las otras patologías los valores estuvieron entre 3.5 y $6.0 \mathrm{~mm}$.

Del total de pacientes incluidas en el estudio solamente cuarenta (40) estaban aún embarazadas en el momento de terminar este; $75 \%$ de ellas cursando la segunda mitad de la gestación y $15 \%$ con embarazo a término.

\section{Conclusiones}

Los resultados obtenidos por el presente estudio no permiten confirmar los reportes existentes en la bibliografía sobre la utilidad de la valoración de ciertos criterios ecográficos en embarazos tempranos mediante ecografía transvaginal y el pronóstico del embarazo, ya que nuestros hallazgos no muestran una correlación estadísticamente significativa que permita hacer predicciones sobre el pronóstico del embarazo tomando como base la cuantificación de la embriocardia, o la medición del saco vitelino o la visualización del cuerpo lúteo.

\section{BIBLIOGRAFIA}

1. Arias F. Guía práctica para el embarazo y el parto de alto riesgo. $2^{\circ} \mathrm{de}$. Mosby/Doyma libros, 1994; 55.

2. Creasy R., Resnik R. Maternal fetal medicine. 3rd. de. W.B. Saunders Company, 1994; 455-457.

3. Levi CS., Lyons EA., Lindsay DJ. Early diagnosis of nonviable pregnancy with endovaginal US. Radiology. 1988; 167: 383-385.

4. Van-Leeuwen Y., Branch DW., Scott JR. First trimester ultrasonography findings in women with a history of recurrent pregnancy loss. Am. J. Obstet. Gynecol. 1993; 168(1 Pt 1): 111-114.

5. Christodoulou $\mathrm{CN}$ et al. Low beta-hCG is associated with poor prognosis in association with an embryo with positive cardiac activity. Ultrasound. Obstet. Gynecol. 1995; 5(4): 267-270.

6. Kurtz AB et al. Can detection of the yolk sac in the first trimester be used to predici the outcome of pregnancy? A prospective sonographic study. Am. J. Roentgenol. 1992; 158 (4): 843-847.

7. Fadda $\mathrm{G}$ et al. Endovaginal ultrasound in patient at risk for repeated abortion. Clin. Exp. Obstet. Gynecol. 1993; 20(2): 120-122.

8. Lindsay DJ et al. Yolk sac diameter and shape at endovaginal U.S: predictors of pregnancy outcome in the first trimester. Radiology. 1992; 183(1): 115-118.

9. Levi CS. Prediction of early pregnancy failure on the basis of mean gestational sac size and absence of a sonographically demostrable yolk sac. Radiology. 1995; 195 (3): 873.

10. Tongsong $\mathrm{T}$ et al. Transvaginal ultrasound in threatened abortions with empty gestational sac. Int. J. Gynaecol. Obstet. 1994; 46(3): 297-301.

11. Britten $\mathrm{S}$ et al. Very early embryonic heart rate in normal pregnancies. Hum-Reprod. 1994; 9(12): 2424-2426.

12. Wisser J., Dirschedl P. Embryonic heart rate in dated human embryos. Early Hum. Dev. 1994; 37(2): 107-115.
13. Benson CB., Doubilet PM. Slow embryonic heart rate in early first trimester: indicator of poor pregnancy outcome. Radiology. 1994; 192(2): 343-344.

14. Glock JL et al. Pronostic significance of morphologic changes of the corpus luteum by transvaginal ultrasound in early pregnancy monitoring. Obstet. Gynecol. 1995; 85(1): 37-41.

15. Zimmer EZ., Chao CR., Santos R. Amniotic sac, fetal heart area, fetal curvature and other morphometrics using first trimester vaginal ultrasonography and color Doppler imaging. J. Ultrasound. Med. 1994; 13(9): 685-690.

16. Tulshinsky D., Hobel CJ. Plasma human chorionic gonadotrophin, estrone, estradiol, progesterone and 17-alfa-hidroxiprogesterone in human pregnancy. Am. J. Obstet. Gynecol. 1973; 117: 884-893.

17. Achiron R., Tadmor O., Mashiach S. Heart rate as a predictor of first trimester spontaneous abortion after ultrasound-proven viability. Obstet. Gynecol. 1991; 78 (3 Pt 1): 330-334.

18. Tadmor, OP et al. Predicting first trimester spontaneous abortion. Ratio of mean sac diameter to crown-rump lenght compared to embryonic heart rate. J. Reprod. Med. 1994; 39(6): 459-462.

19. Daya S. Evaluation of normal gestational sac growth: apperance of embryonic heartbeat and embryo movements using the transvaginal technique (letter). Obstet. Gynecol. 1991; 78(4): 729-730.

20. Daily CA., Laurent SL., Nunley WC (Jr). The pronostic value of serum progesterone and quantitative beta-human chorionic gonadotropin in early human pregnancy. Am. J. Obstet. Gynecol. 1994; 171(2): 380-384.

21. Steiner $\mathrm{H}$ et al. First trimester three dimensional ultrasound volumetry of the gestational sac. Arch. Gynecol. Obstet. 1994; 255(4): 165-170.

22. Ohtsuka-T et al. Relation between gestational sac diameter, crownrump lenght, and maternal serum estradiol, progesterone and prolactin level in early pregnancy. Arch. Gynecol. Obstet. 1995; 256(1): 5-8. 